\title{
STRINGY E-FUNCTIONS OF CANONICAL TORIC FANO THREEFOLDS AND THEIR APPLICATIONS
}

\author{
VICTOR BATYREV AND KARIN SCHALLER
}

\begin{abstract}
Let $\Delta$ be a 3-dimensional lattice polytope containing exactly one interior lattice point. We give a simple combinatorial formula for computing the stringy $E$-function of the 3-dimensional canonical toric Fano variety $X_{\Delta}$ associated with the polytope $\Delta$. Using the stringy Libgober-Wood identity and our formula, we generalize the well-known combinatorial identity $\sum_{\substack{\operatorname{dim}(\theta)=1 \\ \theta \preceq \Delta}} v(\theta) \cdot v\left(\theta^{*}\right)=24$ holding in the case of 3-dimensional reflexive polytopes $\Delta$.
\end{abstract}

\section{INTRODUCTION}

Let $N$ be a free abelian group of rank $d$. A $d$-dimensional convex polytope $\Delta \subseteq$ $N_{\mathbb{R}}:=N \otimes \mathbb{R}$ having vertices in $N$ (i.e., $\Delta=\operatorname{conv}(\Delta \cap N)$ ) is called lattice polytope. A lattice polytope $\Delta$ is called canonical Fano if the origin $0 \in N$ is the only lattice point in its interior $\Delta^{\circ}$, i.e., $\Delta^{\circ} \cap N=\{0\}$. Let $X_{\Delta}$ be the toric variety given by the spanning fan of a canonical Fano polytope $\Delta$, i.e., $X_{\Delta}$ is defined by the fan $\Sigma_{\Delta}:=\left\{\sigma_{\theta} \mid \theta \preceq \Delta\right\}$ whose cones $\sigma_{\theta}:=\mathbb{R}_{\geq 0} \theta$ are spanned by all faces $\theta$ of $\Delta$. It is known that the toric variety $X_{\Delta}$ has at worst canonical singularities [Rei83] and the anticanonical divisor $-K_{X_{\Delta}}$ of $X_{\Delta}$ is an ample $\mathbb{Q}$-Cartier divisor [Dan78], i.e., $X_{\Delta}$ is a canonical toric Fano variety. One can show that any $d$-dimensional canonical toric Fano variety can be obtained from a $d$-dimensional canonical Fano polytope $\Delta$ as above and that there is a bijection between $d$-dimensional canonical toric Fano varieties up to isomorphism and $d$-dimensional canonical Fano polytopes $\Delta \subseteq N_{\mathbb{R}}$ considered up to an isomorphism of the lattice $N$ [Kas10]. The classification of $d$ dimensional canonical toric Fano varieties is known only for $d \leq 3$.

Let $M:=\operatorname{Hom}(N, \mathbb{Z})$ be the dual lattice and $M_{\mathbb{R}}$ the corresponding real vector space together with the natural pairing $\langle\cdot, \cdot\rangle: M_{\mathbb{R}} \times N_{\mathbb{R}} \rightarrow \mathbb{R}$. For any $d$-dimensional convex polytope $\Delta \subseteq N_{\mathbb{R}}$ containing $0 \in N$ in its interior, we consider the dual polytope

$$
\Delta^{*}:=\left\{y \in M_{\mathbb{R}} \mid\langle y, x\rangle \geq-1 \forall x \in \Delta\right\} \subseteq M_{\mathbb{R}} .
$$

There exists a natural bijection (duality) between $k$-dimensional faces $\theta \preceq \Delta$ of $\Delta$ and $(d-k-1)$-dimensional dual faces $\theta^{*} \preceq \Delta^{*}$ of the dual polytope $\Delta^{*}$ defined as

$$
\theta^{*}:=\left\{y \in \Delta^{*} \mid\langle y, x\rangle=-1 \forall x \in \theta\right\} .
$$

A $d$-dimensional lattice polytope $\Delta \subseteq N_{\mathbb{R}}$ containing $0 \in N$ in its interior is called reflexive if all vertices of the dual polytope $\Delta^{*}$ belong to the dual lattice $M$, i.e., if $\Delta^{*}$ is also a lattice polytope [Bat94]. If $\Delta$ is reflexive, then $\Delta^{*}$ is also reflexive and 
$\left(\Delta^{*}\right)^{*}=\Delta$. Any facet $\theta \preceq \Delta$ of a reflexive polytope $\Delta$ is defined by an equation $\langle m, x\rangle=-1$ for some lattice vertex $m \in M$ of the dual reflexive polytope $\Delta^{*}$. This means that each facet $\theta$ of a reflexive polytope $\Delta$ has lattice distance 1 to the origin $0 \in N$ and the origin is the only interior lattice point of $\Delta$. In particular, every reflexive polytope is a canonical Fano polytope. The converse is not true. If $\Delta$ is a canonical Fano polytope, then the lattice distance $n_{\theta}$ from a $(d-1)$-dimensional face $\theta \preceq \Delta$ to the origin can be larger than 1 , i.e., the facet $\theta$ is defined by an equation $-\langle m, x\rangle=n_{\theta}>1$ for some primitive lattice point $m \in M$. One can show that any canonical Fano polytope of dimension $d \leq 2$ is always reflexive, but the latter is not true if $d \geq 3$. There exist exactly 4,319 isomorphism classes of 3-dimensional reflexive polytopes classified by Kreuzer and Skarke [KS98]. However, there exist much more (exactly 674,688) isomorphism classes of 3-dimensional canonical Fano polytopes classified by Kasprzyk [Kas10].

If $\Delta \subseteq N_{\mathbb{R}}$ is an arbitrary $d$-dimensional lattice polytope, then we define $v(\Delta)$ to be the normalized volume, i.e., the positive integer $d ! \cdot \operatorname{vol}_{d}(\Delta)$, where $\operatorname{vol}_{d}(\Delta)$ denotes the $d$-dimensional volume of $\Delta$ with respect to the lattice $N$. Similarly, we define the positive integer $v(\theta):=k ! \cdot \operatorname{vol}_{k}(\theta)$ for a $k$-dimensional face $\theta \preceq \Delta$, where $\operatorname{vol}_{k}(\theta)$ denotes the $k$-dimensional volume of $\theta$ with respect to the sublattice $\operatorname{span}(\theta) \cap N$. If $\Delta \subseteq N_{\mathbb{R}}$ is a $d$-dimensional polytope having vertices in $N_{\mathbb{Q}}:=N \otimes \mathbb{Q}$, i.e., $\Delta$ is a rational polytope, then we can similarly define the positive rational number $v(\theta)$ for any $k$-dimensional face $\theta \preceq \Delta$. For this purpose, we consider an integer $l$ such that $l \Delta$ is a lattice polytope and define for a $k$-dimensional face $\theta \preceq \Delta$ its volume as $v(\theta):=\frac{1}{l^{k}} v(l \theta)$.

For any 3-dimensional reflexive polytope $\Delta$ one has the well-known combinatorial identity

$$
24=\sum_{\substack{\theta \preceq \Delta \\ \operatorname{dim}(\theta)=1}} v(\theta) \cdot v\left(\theta^{*}\right)
$$

$\left[\mathrm{BCF}^{+} 05\right.$, Theorem 4.3] (Corollary 1.8). A possible proof of this identity can be obtained from the fact that the Euler number of a smooth $K 3$-surface equals 24 . In addition, one considers a generic affine hypersurface $Z_{\Delta} \subseteq\left(\mathbb{C}^{*}\right)^{3}$ defined by a Laurent polynomial $g_{\Delta} \in \mathbb{C}\left[x_{1}^{ \pm 1}, x_{2}^{ \pm 1}, x_{3}^{ \pm 1}\right]$ with the reflexive Newton polytope $\Delta$ in the 3-dimensional torus $\left(\mathbb{C}^{*}\right)^{3} \subseteq X_{\Delta^{*}}$ and applies the following two statements:

- The projective closure $\overline{Z_{\Delta}} \subseteq X_{\Delta^{*}}$ of $Z_{\Delta}$ is a $K 3$-surface with at worst canonical singularities [Bat94, Theorem 4.1.9];

- The number

$$
\sum_{\substack{\theta \preceq \Delta \\ \operatorname{dim}(\theta)=1}} v(\theta) \cdot v\left(\theta^{*}\right)
$$

equals the stringy Euler number of $\overline{Z_{\Delta}}$ [BD96, Corollary 7.10].

One of our results is a generalization of the combinatorial identity $(\star)$ for arbitrary 3 -dimensional canonical Fano polytopes $\Delta$. First, we consider a 3 -dimensional lattice 
polytope $\Delta \subseteq N_{\mathbb{R}}$ such that a generic affine hypersurface $Z_{\Delta} \subseteq\left(\mathbb{C}^{*}\right)^{3}$ defined by a Laurent polynomial $f_{\Delta} \in \mathbb{C}\left[x_{1}^{ \pm 1}, x_{2}^{ \pm 1}, x_{3}^{ \pm 1}\right]$ with the Newton polytope $\Delta$ is birational to a smooth $K 3$-surface. Such a lattice polytope $\Delta$ must be a 3 -dimensional canonical Fano polytope, because the number of interior lattice points in $\Delta$ equals the geometric genus of the affine surface $Z_{\Delta} \subseteq\left(\mathbb{C}^{*}\right)^{3}[$ Kho78] and it equals 1 for a smooth $K 3$ surface. Moreover, one can show that the above condition on $\Delta$ is satisfied if and only if $\Delta$ is contained in some reflexive polytope $\Delta^{\prime}$. Those 3-dimensional canonical Fano polytopes are called almost reflexive. There exist many equivalent characterizations of 3-dimensional almost reflexive polytopes $\Delta$ [Bat17], e.g., $\Delta \subseteq N_{\mathbb{R}}$ is almost reflexive if and only if the convex hull $\left[\Delta^{*}\right]:=\operatorname{conv}\left(\Delta^{*} \cap M\right)$ of all lattice points in the dual polytope $\Delta^{*} \subseteq M_{\mathbb{R}}$ is a reflexive polytope. Using the list of all 3-dimensional canonical Fano polytopes [Kas10], Kasprzyk showed that among all 674,688 3-dimensional canonical Fano polytopes there exist exactly 9,089 lattice polytopes $\Delta$ which are not almost reflexive. In other words, there exist exactly 665,599 almost reflexive polytopes of dimension 3 . Any almost reflexive polytope $\Delta$ allows us to construct a family of smooth $K 3$-surfaces using the toric variety $X^{\Delta}$ associated with the normal fan $\Sigma^{\Delta}$ of $\Delta$. We recall that the normal fan $\Sigma^{\Delta}:=\left\{\sigma^{\theta} \mid \theta \preceq \Delta\right\}$ consists of cones $\sigma^{\theta}:=\mathbb{R}_{\geq 0} \theta^{*}$ corresponding to faces $\theta \preceq \Delta$, where $\sigma^{\theta}$ is the cone generated by the dual face $\theta^{*} \preceq \Delta^{*}$ of the dual polytope $\Delta^{*}$ and $\operatorname{dim}\left(\sigma^{\theta}\right)=\operatorname{dim}\left(\theta^{*}\right)+1=d-\operatorname{dim}(\theta)$.

A generalization of the combinatorial formula $(\star)$ for an arbitrary 3-dimensional almost reflexive polytope $\Delta$ is the following statement:

Theorem A. Let $\Delta \subseteq N_{\mathbb{R}}$ be an arbitrary 3-dimensional almost reflexive polytope. Then

$$
24=v(\Delta)-\sum_{\substack{\theta \preceq \Delta \\ \operatorname{dim}(\theta)=2}} \frac{1}{n_{\theta}} \cdot v(\theta)+\sum_{\substack{\theta \preceq \Delta \\ \operatorname{dim}(\theta)=1}} v(\theta) \cdot v\left(\theta^{*}\right) .
$$

The proof of this new combinatorial formula is built around the more general combinatorial formula

$$
e_{\mathrm{str}}(Y)=\sum_{\substack{\theta \preceq \Delta \\ \operatorname{dim}(\theta) \geq 1}}(-1)^{\operatorname{dim}(\theta)-1} v(\theta) \cdot v\left(\sigma^{\theta} \cap \Delta^{*}\right)
$$

[Bat17, Theorem 4.11] (Theorem 1.7) computing the stringy Euler number $e_{\text {str }}(Y)$ of a $(d-1)$-dimensional Calabi-Yau variety $Y$ with at worst canonical singularities which is birational to a generic $(d-1)$-dimensional affine hypersuface $Z_{\Delta} \subseteq\left(\mathbb{C}^{*}\right)^{d}$ defined by a generic Laurent polynomial $f_{\Delta} \in \mathbb{C}\left[x_{1}^{ \pm 1}, \cdots, x_{d}^{ \pm 1}\right]$ with the Newton polytope $\Delta$. If $d=3$, as we mentioned, such a $(d-1)$-dimensional Calabi-Yau variety $Y$ exists if and only if $\Delta$ is a 3 -dimensional almost reflexive polytope.

It is remarkable that the same identity holds for all 9,089 lattice polytopes $\Delta$ which are not almost reflexive, i.e., it holds for all 674,688 3-dimensional canonical Fano polytopes $\Delta$. 
Theorem B. Let $\Delta \subseteq N_{\mathbb{R}}$ be an arbitrary 3-dimensional canonical Fano polytope. Then

$$
24=v(\Delta)-\sum_{\substack{\theta \preceq \Delta \\ \operatorname{dim}(\theta)=2}} \frac{1}{n_{\theta}} \cdot v(\theta)+\sum_{\substack{\theta \preceq \Delta \\ \operatorname{dim}(\theta)=1}} v(\theta) \cdot v\left(\theta^{*}\right) .
$$

The proof of this more general statement uses a combinatorial formula for computing the stringy $E$-function $E_{\text {str }}\left(X_{\Delta} ; u, v\right)$ of a 3-dimensional canonical toric Fano variety $X_{\Delta}$.

Theorem C. Let $X_{\Delta}$ be a 3-dimensional canonical toric Fano variety defined by a 3-dimensional canonical Fano polytope $\Delta \subseteq N_{\mathbb{R}}$. Then the stringy E-function of $X_{\Delta}$ can be computed as

$$
E_{\mathrm{str}}\left(X_{\Delta} ; u, v\right)=\left((u v)^{3}+1\right)+r \cdot\left((u v)^{2}+(u v)\right)+\sum_{\substack{\theta \leq \Delta \\ \operatorname{dim}(\theta)=2, n_{\theta}>1}} v(\theta) \cdot\left(\sum_{k=1}^{n_{\theta}-1}(u v)^{\frac{k}{n_{\theta}}+1}\right),
$$

where $r:=|\Delta \cap N|-4$.

In order to prove Theorem B, we combine the above formula for the stringy $E$ function $E_{\text {str }}\left(X_{\Delta} ; u, v\right)$ with our combinatorial version of the stringy Libgober-Wood identity in terms of generalized stringy Hodge numbers and intersection products of stringy Chern classes [BS17, Theorem 4.3].

Organisation of the paper. In Section 1, we launch remaining and necessary definitions as well as explain important relationships focused on almost reflexive polytopes. In addition, we prove Theorem A.

In Section 2, we investigate the stringy $E$-function of arbitrary 3-dimensional toric Fano varieties with at worst canonical singularities and prove Theorem $\mathrm{C}$.

In Section 3, we apply Theorem C in order to prove Theorem B using in addition a combinatorial version of the stringy Libgober-Wood identity.

Acknowledgments. We would like to thank Tom Coates, Alessio Corti, Alexander Kasprzyk, and Hannah Markwig for inspiring discussions.

\section{Stringy Euler numbers and Calabi-Yau hypersurfaces}

The orbifold or stringy Euler number has been introduced by Dixon, Harvey, Vafa, and Witten [DHVW85] as a natural topological invariant motivated by string theory for singular Calabi-Yau varieties. Further investigations of mirror symmetry for singular Calabi-Yau varieties led to the notion of stringy $E$-functions [Bat98]. We recall their definition below.

Let $X$ be a $d$-dimensional normal projective $\mathbb{Q}$-Gorenstein variety with at worst log-terminal singularities, i.e., the canonical divisor $K_{X}$ of $X$ is a $\mathbb{Q}$-Cartier divisor and for some desingularization $\rho: X^{\prime} \rightarrow X$ of $X$, whose exceptional locus is a union of smooth irreducible divisors $D_{1}, \ldots, D_{s}$ with only normal crossings, one has $K_{X^{\prime}}=$ $\rho^{*} K_{X}+\sum_{i=1}^{s} a_{i} D_{i}$ for some rational numbers $a_{i}>-1$ for all $1 \leq i \leq s$. Moreover, we 
define $D_{J}$ to be the intersection $\cap_{j \in J} D_{j}$ for any nonempty subset $J \subseteq I:=\{1, \ldots, s\}$ and set $D_{\emptyset}:=X^{\prime}$. We remark that the subvariety $D_{J} \subseteq X^{\prime}$ is either empty or a smooth projective subvariety of $X^{\prime}$ of codimension $|J|$. The stringy E-function of $X$ is a rational algebraic function in two variables $u, v$ defined as

$$
E_{\mathrm{str}}(X ; u, v):=\sum_{\emptyset \subseteq J \subseteq I} E\left(D_{J} ; u, v\right) \prod_{j \in J}\left(\frac{u v-1}{(u v)^{a_{j}+1}-1}-1\right)
$$

[Bat98, Definition 2.1], where $E\left(D_{J} ; u, v\right)=\sum_{0 \leq p, q \leq d-|J|}(-1)^{p+q} h^{p, q}\left(D_{J}\right) u^{p} v^{q}$ is the generating polynomial for the Hodge numbers $h^{p, q}\left(D_{J}\right)$ of the smooth projective variety $D_{J}$. The stringy Euler number of $X$ is defined as

$$
e_{\mathrm{str}}(X):=\lim _{u, v \rightarrow 1} E_{\mathrm{str}}(X ; u, v)=\sum_{\emptyset \subseteq J \subseteq I} c_{d-|J|}\left(D_{J}\right) \prod_{j \in J}\left(\frac{-a_{j}}{a_{j}+1}\right) .
$$

The restriction on the singularities of $X$ is used to proof the independence of $E_{\text {str }}(X ; u$, $v$ ) on the choice of the desingularization $\rho$ [Bat98, Theorem 3.4]. As a special case, this formula implies the equality $E_{\text {str }}(X ; u, v)=E(X ; u, v)$ if $X$ is smooth and the equality $E_{\text {str }}(X ; u, v)=E\left(X^{\prime} ; u, v\right)$ if $\rho: X^{\prime} \rightarrow X$ is a crepant desingularization of $X$, i.e., if all rational numbers $a_{i}$ are zero.

It is very convenient to consider stringy invariants while investigating singular varieties $X$ that appear in birational geometry and the minimal model program. It is known that a minimal model $X^{\prime}$ of a projective algebraic variety $X$ with at worst log-terminal singularities is not uniquely determined, because sometimes there exists another minimal model $X^{\prime \prime}$ of $X$, which is obtained from $X^{\prime}$ by a sequence of flops. Nevertheless, the stringy $E$-functions of $X^{\prime}$ and $X^{\prime \prime}$ are the same, i.e., $E_{\mathrm{str}}\left(X^{\prime} ; u, v\right)=$ $E_{\text {str }}\left(X^{\prime \prime} ; u, v\right)$. The same equality $E_{\mathrm{str}}\left(X^{\prime} ; u, v\right)=E_{\mathrm{str}}\left(X^{\prime \prime} ; u, v\right)$ holds if the minimal model $X^{\prime}$ admits a crepant birational morphism $X^{\prime} \rightarrow X^{\prime \prime}$ to a projective variety $X^{\prime \prime}$ with at worst canonical $\mathbb{Q}$-Gorenstein singularities. In particular, the stringy $E$ function of a minimal birational model of $X$ and the stringy $E$-function of a canonical model of $X$ are the same, i.e., they are independent of the choice of these birational models.

In this section, we are interested in the stringy Euler numbers $e_{\mathrm{str}}(Y)$ of minimal and canonical models of algebraic varieties $Y$ that are birational to a generic affine hypersurface $Z_{\Delta} \subseteq\left(\mathbb{C}^{*}\right)^{d}$ defined by a generic Laurent polynomial $f_{\Delta} \in \mathbb{C}\left[x_{1}^{ \pm 1}, \ldots, x_{d}^{ \pm 1}\right]$ whose Newton polytope is a given $d$-dimensional lattice polytope $\Delta \subseteq N_{\mathbb{R}}$. In this case, the stringy Euler number $e_{\text {str }}(Y)$ is uniquely determined by the lattice polytope $\Delta$ and it can be computed by a combinatorial formula. The generic open condition on the coefficients of the Laurent polynomial $f_{\Delta}$ can be formulated in a more precise form:

Definition 1.1. Let $\Delta \subseteq N_{\mathbb{R}}$ be a $d$-dimensional lattice polytope and $Z_{\Delta} \subseteq \mathbb{T}_{d} \cong$ $\left(\mathbb{C}^{*}\right)^{d}$ an affine hypersurface, which is the zero set of a Laurent polynomial

$$
f_{\Delta}(x)=\sum_{n \in \Delta \cap \mathbb{Z}^{d}} a_{n} x^{n} \in \mathbb{C}\left[x_{1}^{ \pm 1}, \ldots, x_{d}^{ \pm 1}\right]
$$


with the Newton polytope $\Delta$ and some sufficiently general coefficients $a_{n} \in \mathbb{C}$. The Laurent polynomial $f_{\Delta}$ and the affine hypersurface $Z_{\Delta}$ are called $\Delta$-nondegenerate if for every face $\theta \preceq \Delta$ the zero locus $Z_{\theta}:=\left\{x \in \mathbb{T}_{d} \mid f_{\theta}(x)=0\right\}$ of the $\theta$-part of $f_{\Delta}$ is empty or a smooth affine hypersurface in the $d$-dimensional algebraic torus $\mathbb{T}_{d}$.

Definition 1.2. Let $\Delta \subseteq N_{\mathbb{R}}$ be a $d$-dimensional lattice polytope containing the origin $0 \in N$ in its interior. The lattice polytope $\Delta$ is called almost pseudoreflexive if the convex hull $\left[\Delta^{*}\right]=\operatorname{conv}\left(\Delta^{*} \cap M\right)$ of all lattice points in the dual polytope $\Delta^{*}$ also contains the origin $0 \in M$ in its interior. An almost pseudoreflexive polytope $\Delta$ is called pseudoreflexive if $\Delta=\left[\left[\Delta^{*}\right]^{*}\right]$. If $\Delta$ is an almost pseudoreflexive polytope, then $\left[\Delta^{*}\right]$ is always a pseudoreflexive polytope. We call an almost pseudoreflexive polytope $\Delta$ almost reflexive if $\left[\Delta^{*}\right]$ is a reflexive polytope.

Using a result of Skarke [Ska96], one obtains that every pseudoreflexive polytope $\Delta$ of dimension $d \leq 4$ is reflexive. In particular, every almost pseudoreflexive polytope of dimension $d \leq 4$ is almost reflexive. There exists an equivalent description of almost reflexive polytopes $\Delta$ of dimension $d \leq 4: \Delta$ is almost pseudoreflexive if and only if $\Delta$ contains $0 \in N$ in its interior and is contained in some reflexive polytope $\Delta^{\prime}$ [Bat17, Proposition 3.4 and Theorem 3.12].

The above classes of lattice polytopes $\Delta$ are related to some (singular) Calabi-Yau varieties:

Definition 1.3. Let $Y$ be a $(d-1)$-dimensional normal irreducible projective algebraic variety with trivial canonical divisor $K_{Y}$. We call $Y$ a Calabi-Yau variety if $Y$ has at worst canonical Gorenstein singularities and $H^{i}\left(Y, \mathcal{O}_{Y}\right)=0(0<i<d-1)$.

Almost pseudoreflexive polytopes $\Delta$ of dimension $d$ are in the following sense closely related to Calabi-Yau varieties:

Proposition 1.4 ([Bat17, Theorem 2.23]). Let $\Delta \subseteq N_{\mathbb{R}}$ be a d-dimensional lattice polytope containing the origin $0 \in N$ in its interior. A $\Delta$-nondegenerate affine hypersurface $Z_{\Delta} \subseteq \mathbb{T}^{d}$ is birational to a $(d-1)$-dimensional Calabi-Yau variety $Y$ if and only the polytope $\Delta$ is almost pseudoreflexive. In particular, in the case $d=3$ a $\Delta$-nondegenerated affine surface $Z_{\Delta} \subseteq \mathbb{T}^{3}$ is birational to a K3-surface if and only if $\Delta$ is an almost reflexive polytope.

In this paper, we consider the following three running examples of 3-dimensional canonical Fano polytopes $\Delta_{1}, \Delta_{2}$, and $\Delta_{3}$ :

Example 1.5. Set $\Delta_{1}:=\operatorname{conv}\left(e_{1}, e_{2}, e_{3},-e_{1}-e_{2}-e_{3}\right), \Delta_{2}:=\operatorname{conv}\left(e_{1}, e_{2}, e_{3},-e_{1}-\right.$ $\left.e_{2}-2 e_{3}\right)$, and $\Delta_{3}:=\operatorname{conv}\left(e_{1}, e_{2}, e_{3},-5 e_{1}-6 e_{2}-8 e_{3}\right)$ (Figure 1). All these polytopes contain the origin $0 \in \mathbb{Z}^{3}$ as an unique interior lattice point. The canonical Fano polytope $\Delta_{1}$ is reflexive, but the canonical Fano polytopes $\Delta_{2}$ and $\Delta_{3}$ are not reflexive. One can check that the lattice polytope $\left[\Delta_{i}^{*}\right]=\operatorname{conv}\left(\Delta_{i}^{*} \cap N\right)$ contains the origin in its interior (Figure 2,3, and 8) if and only if $i \in\{1,2\}$. So the lattice simplices $\Delta_{1}$, $\Delta_{2}$ are almost reflexive, but the lattice simplex $\Delta_{3}$ is not almost reflexive. 


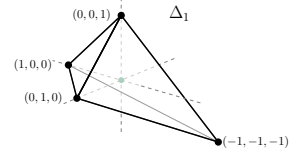

(a) $\Delta_{1}$ reflexive.

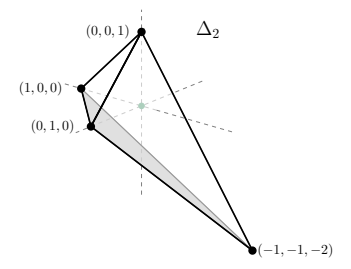

(b) $\Delta_{2}$ almost reflexive.

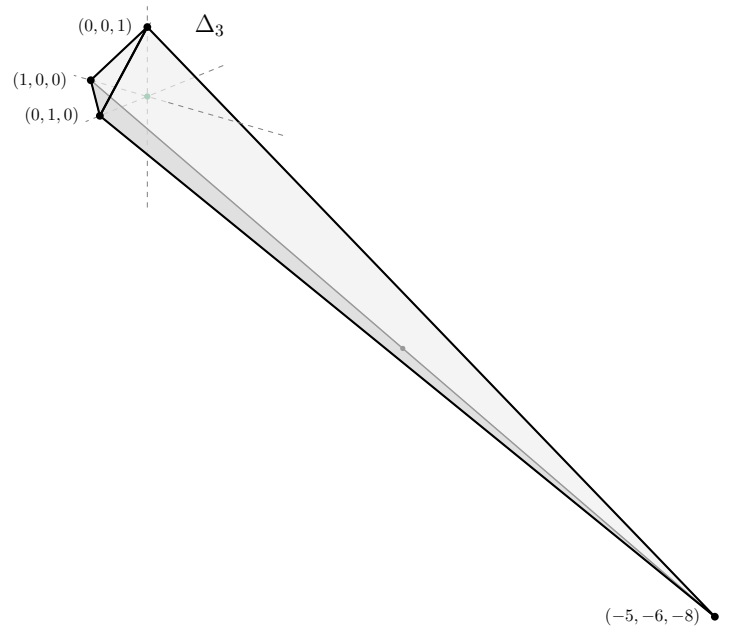

(c) $\Delta_{3}$ not almost reflexive.

FiguRE 1. Three types of canonical Fano polytopes in dimension 3.

Now we formulate a main result of [Bat17]:

Theorem 1.6 ([Bat17, Theorem 4.11]). Let $\Delta \subseteq N_{\mathbb{R}}$ be an arbitrary d-dimensional almost pseudoreflexive polytope. Denote by $Y$ a canonical Calabi-Yau model of a $\Delta$ nondegenerate affine hypersurface $Z_{\Delta} \subseteq \mathbb{T}_{d}$. Then the stringy Euler number of $Y$ can be computed by the combinatorial formula

$$
e_{\mathrm{str}}(Y)=\sum_{\substack{\theta \preceq \Delta \\ \operatorname{dim}(\theta) \geq 1}}(-1)^{\operatorname{dim}(\theta)-1} v(\theta) \cdot v\left(\sigma^{\theta} \cap \Delta^{*}\right),
$$

where $\sigma^{\theta}=\mathbb{R}_{\geq 0} \theta^{*}$ is the cone in the normal fan $\Sigma^{\Delta}$ of $\Delta$ corresponding to the dual face $\theta^{*} \preceq \Delta^{*}$ of the dual polytope $\Delta^{*} \subseteq M_{\mathbb{R}}$.

The combinatorial formula for the stringy Euler number in Theorem 1.6 can be rewritten in the following equivalent form:

Theorem 1.7. Let $\Delta \subseteq N_{\mathbb{R}}$ be an arbitrary d-dimensional almost pseudoreflexive polytope. Denote by $Y$ a canonical Calabi-Yau model of a $\Delta$-nondegenerated affine hypersurface $Z_{\Delta} \subseteq \mathbb{T}_{d}$. Then the stringy Euler number of $Y$ can be computed by the combinatorial formula

$$
e_{\text {str }}(Y)=v(\Delta)-\sum_{\substack{\theta \leq \Delta \\ \operatorname{dim}(\theta)=d-1}} \frac{1}{n_{\theta}} \cdot v(\theta)+\sum_{\substack{\theta \leq \Delta \\ 1 \leq \operatorname{dim}(\theta) \leq d-2}}(-1)^{\operatorname{dim}(\theta)-1} v(\theta) \cdot v\left(\theta^{*}\right) .
$$

Proof. Let $\theta \preceq \Delta$ be a face of $\Delta$. If $\theta=\Delta$, then $\sigma^{\theta} \cap \Delta^{*}=\{0\}$ and $v\left(\sigma^{\theta} \cap \Delta^{*}\right)=1$. If $\theta$ is a $(d-1)$-dimensional face of $\Delta$ and $u_{\theta}$ denotes the primitive inward-pointing facet normal of $\theta$, then $\sigma^{\theta} \cap \Delta^{*}=\operatorname{conv}\left(0,1 / n_{\theta} \cdot u_{\theta}\right)$ and $v\left(\sigma^{\theta} \cap \Delta^{*}\right)=1 / n_{\theta}$. If $\theta \preceq \Delta$ is face of $\Delta$ of dimension $k(1 \leq k \leq d-2)$, then $\sigma^{\theta} \cap \Delta^{*}$ is a $(d-k)$-dimensional pyramid with vertex $0 \in M$ over the $(d-k-1)$-dimensional dual face $\theta^{*}$. By definition of 
the dual polytope $\Delta^{*}$, the lattice distance between 0 and $\theta^{*}$ equals 1 . So we get $v\left(\sigma^{\theta} \cap \Delta^{*}\right)=v\left(\theta^{*}\right)$. Now it remains to apply the formula of Theorem 1.6.

We apply this theorem in the case $d=3$ to get a new combinatorial identity for the Euler number 24:

Theorem A. Let $\Delta \subseteq N_{\mathbb{R}}$ be an arbitrary 3-dimensional almost reflexive polytope. Then

$$
24=v(\Delta)-\sum_{\substack{\theta \leq \Delta \\ \operatorname{dim}(\theta)=2}} \frac{1}{n_{\theta}} \cdot v(\theta)+\sum_{\substack{\theta \preceq \Delta \\ \operatorname{dim}(\theta)=1}} v(\theta) \cdot v\left(\theta^{*}\right) .
$$

Proof. A canonical Calabi-Yau model $Y$ of a $\Delta$-nondegenerate affine hypersurface $Z_{\Delta} \subseteq \mathbb{T}_{3}$ is a $K 3$-surface with at worst canonical singularities (Proposition 1.4). Its minimal crepant desingularization $\tilde{Y}$ is a smooth $K 3$-surface and by Noether's Theorem

$$
2=\chi\left(\tilde{Y}, \mathcal{O}_{\tilde{Y}}\right)=\frac{1}{12}\left(c_{1}(\tilde{Y})^{2}+c_{2}(\tilde{Y})\right)
$$

the stringy Euler number $e_{\mathrm{str}}(Y)$ equals $c_{2}(\tilde{Y})=24$. Using the formula for $e_{\text {str }}(Y)$ from Theorem 1.7, we get the required identity.

The statement of Theorem A specializes to the well-known formula for reflexive polytopes in dimension $3\left[\mathrm{BCF}^{+} 05\right.$, Theorem 4.3] (Equation $\left.(\star)\right)$ :

Corollary 1.8. Let $\Delta \subseteq N_{\mathbb{R}}$ be a 3-dimensional reflexive polytope. Then

$$
24=\sum_{\substack{\theta \preceq \Delta \\ \operatorname{dim}(\theta)=1}} v(\theta) \cdot v\left(\theta^{*}\right) .
$$

Proof. We apply Theorem A and get

$$
24=v(\Delta)-\sum_{\substack{\theta \preceq \Delta \\ \operatorname{dim}(\theta)=2}} \frac{1}{n_{\theta}} \cdot v(\theta)+\sum_{\substack{\theta \leq \Delta \\ \operatorname{dim}(\theta)=1}} v(\theta) \cdot v\left(\theta^{*}\right) .
$$

Since $\Delta$ is reflexive, we have $n_{\theta}=1$ for all facets $\theta \preceq \Delta$ and

$$
v(\Delta)-\sum_{\substack{\theta \preceq \Delta \\ \operatorname{dim}(\theta)=2}} \frac{1}{n_{\theta}} \cdot v(\theta)=v(\Delta)-\sum_{\substack{\theta \preceq \Delta \\ \operatorname{dim}(\theta)=2}} v(\theta)=0 .
$$

The equation from above simplifies to

$$
24=\sum_{\substack{\theta \preceq \Delta \\ \operatorname{dim}(\theta)=1}} v(\theta) \cdot v\left(\theta^{*}\right) .
$$

Example 1.9. For the reflexive polytope $\Delta_{1}=\operatorname{conv}\left(e_{1}, e_{2}, e_{3},-e_{1}-e_{2}-e_{3}\right)$ (Figure 2(a) and 1(a)) we get the identity

$$
\sum_{\substack{\theta \leq \Delta_{1} \\ \operatorname{dim}(\theta)=1}} v(\theta) \cdot v\left(\theta^{*}\right)=6 \cdot(1 ! \cdot 1) \cdot(1 ! \cdot 4)=24,
$$


because the dual polytope equals

$$
\Delta_{1}^{*}=\operatorname{conv}((3,-1,-1),(-1,3,-1),(-1,-1,3),(-1,-1,-1))
$$

and $\operatorname{dim}\left(\theta^{*}\right)=1$ with $v\left(\theta^{*}\right)=1$ ! $\cdot 4=4$ for every 1 -dimensional face $\theta \preceq \Delta_{1}$.

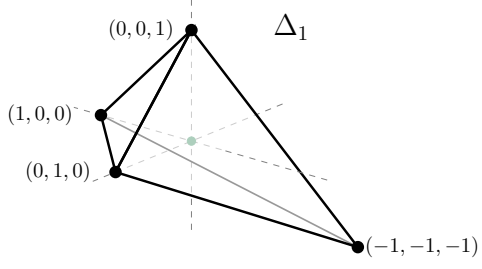

(a)

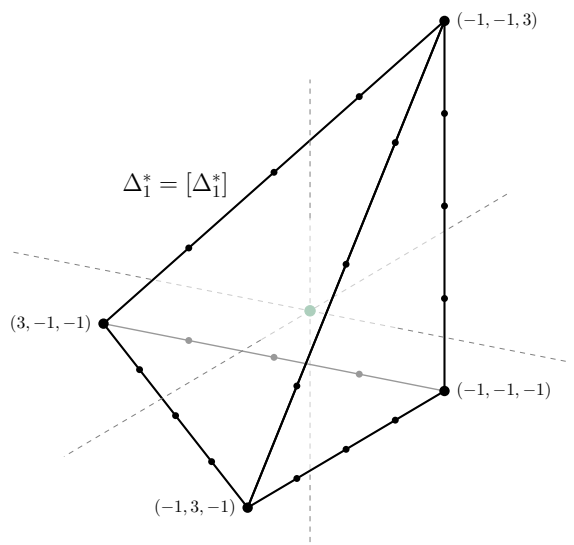

(b)

FiguRE 2. Illustration of a reflexive polytope.

Example 1.10. Consider the almost reflexive polytope $\Delta_{2}=\operatorname{conv}\left(e_{1}, e_{2}, e_{3},-e_{1}-\right.$ $e_{2}-2 e_{3}$ ) (Figure $3(\mathrm{a})$ and $1(\mathrm{~b})$ ). Its dual polytope $\Delta_{2}^{*}$ is rational:

$$
\Delta_{2}^{*}=\operatorname{conv}((4,-1,-1),(-1,4,-1),(-1,-1,3 / 2),(-1,-1,-1)) .
$$

There exists exactly one 2-dimensional face (highlighted in gray in Figure $3(\mathrm{a})$ ) of $\Delta_{2}$ having lattice distance $2>1$ to the origin. The combinatorial identity from Theorem A holds, because

$$
\begin{aligned}
& v\left(\Delta_{2}\right)-\sum_{\substack{\theta \preceq \Delta_{2} \\
\operatorname{dim}(\theta)=2}} \frac{1}{n_{\theta}} \cdot v(\theta)+\sum_{\substack{\theta \preceq \Delta_{2} \\
\operatorname{dim}(\theta)=1}} v(\theta) \cdot v\left(\theta^{*}\right) \\
& =5-\left(\frac{1}{2} \cdot 1+1 \cdot 1+1 \cdot 1+1 \cdot 1\right)+\left(1 \cdot \frac{5}{2}+1 \cdot \frac{5}{2}+1 \cdot \frac{5}{2}+1 \cdot 5+1 \cdot 5+1 \cdot 5\right) \\
& =5-3.5+22.5=24 .
\end{aligned}
$$

\section{Stringy E-FUnCtions of CANONiCAL toric FAnO threEFolds}

Let $X$ be a $d$-dimensional projective $\mathbb{Q}$-Gorenstein toric variety associated with a fan $\Sigma$ of rational polyhedral cones in $N_{\mathbb{R}}$. We denote by $\Sigma(k)$ the set of all $k$ dimensional cones in $\Sigma$. We choose $\Sigma^{\prime}$ to be a simplicial subdivision of the fan $\Sigma$ such that $\Sigma^{\prime}(1) \subseteq \Sigma(1)$ and define $\kappa_{\Sigma}$ to be the $\Sigma$-piecewise linear function corresponding to the anticanonical divisor $-K_{X}$ of $X$ (i.e., $\kappa_{\Sigma}$ has value -1 on every primitive ray generator of a 1-dimensional cone $\rho \in \Sigma(1))$. Moreover, we define $\square_{\sigma}^{\circ}:=\sigma^{\circ} \cap \square_{\sigma}$ for any cone $\sigma \in \Sigma^{\prime}$, where $\sigma^{\circ}$ denotes the relative interior of $\sigma$ and $\square_{\sigma}$ the parallelepiped spanned by the primitive ray generators of the cone $\sigma$. 


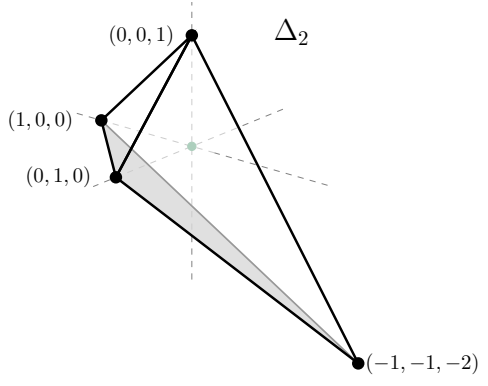

(a)

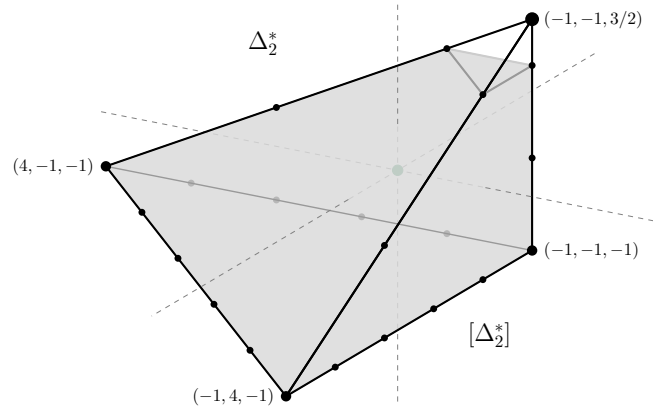

(b)

FIGURE 3. Illustration of an almost reflexive polytope.

There is a general formula for the stringy $E$-function $E_{\mathrm{str}}(X ; u, v)$ of the $d$-dimensional projective $\mathbb{Q}$-Gorenstein toric variety $X$ :

Proposition 2.1 ([BS17, Proposition 4.1]). Let $X$ be a d-dimensional projective $\mathbb{Q}$ Gorenstein toric variety associated with a fan $\Sigma$. Then the stringy E-function of $X$ can be computed as the finite sum

$$
E_{\mathrm{str}}(X ; u, v)=\sum_{\sigma \in \Sigma^{\prime}}(u v-1)^{d-\operatorname{dim}(\sigma)} \sum_{n \in \square_{\sigma}^{\circ} \cap N}(u v)^{\operatorname{dim}(\sigma)+\kappa_{\Sigma}(n)} .
$$

In particular,

$$
E_{\text {str }}(X ; u, v)=\sum_{\alpha} \psi_{\alpha}(\Sigma)(u v)^{\alpha}
$$

for some nonnegative integers $\psi_{\alpha}(\Sigma)$ and some nonnegative rational numbers $\alpha$.

A much more precise computation of the stringy $E$-function $E_{\text {str }}(X ; u, v)$ has been obtained if $X$ is a toric log del Pezzo surface, i.e., a normal projective toric surface with at worst log-terminal singularities and an ample anticanonical $\mathbb{Q}$-Cartier divisor $-K_{X}$ [KKN10]. Such a toric surface is defined by a LDP-polygon $\Delta \subseteq N_{\mathbb{R}}$, i.e., a 2-dimensional lattice polytope containing the origin $0 \in N$ in its interior such that all vertices of $\Delta$ are primitive lattice points in $N$. In this case, $\Sigma=\Sigma_{\Delta}$ is the spanning fan in the 2-dimensional space $N_{\mathbb{R}}$ consisting of cones over faces of the $L D P$-polygon $\Delta$. Using Proposition 2.1, we got

Theorem 2.2 ([BS17, Corollary 4.5]). Let $X$ be a toric log del Pezzo surface defined by a LDP-polygon $\Delta \subseteq N_{\mathbb{R}}$. Then the stringy E-function of $X$ can be computed as

$$
E_{\text {str }}(X ; u, v)=\left((u v)^{2}+1\right)+r \cdot(u v)+\sum_{n \in \Delta^{\circ} \backslash\{0\}}\left((u v)^{2+\kappa_{\Delta}(n)}+(u v)^{-\kappa_{\Delta}(n)}\right),
$$

where $r:=|\partial \Delta \cap N|-2$ and $\kappa_{\Delta}$ is the $\Sigma_{\Delta}$-piecewise linear function corresponding to the anticanonical divisor $-K_{X}$ of $X$ with value -1 on the whole boundary $\partial \Delta$ of $\Delta$.

Example 2.3. Set $\Delta:=\operatorname{conv}\left(e_{1}, e_{2},-e_{1}-e_{2}\right)$ and $\Delta_{m}:=\operatorname{conv}\left(e_{1}, e_{2},-e_{1}-m e_{2}\right)$ for an integer $m \in \mathbb{N}$ (Figure $4\left(\right.$ a) and $4(\mathrm{~b})$ ), i.e., $\Delta$ is a reflexive polygon and $\Delta_{m}$ a 
$L D P$-polygon. Therefore, $X_{\Delta}=\mathbb{P}_{2}$ and $X_{\Delta_{m}}$ are toric log del Pezzo surfaces with

$$
E_{\mathrm{str}}\left(X_{\Delta} ; u, v\right)=(u v)^{2}+(u v)+1
$$

and

$$
E_{\mathrm{str}}\left(X_{\Delta_{3}} ; u, v\right)=\left((u v)^{2}+1\right)+(u v)+\left((u v)^{\frac{4}{3}}+(u v)^{\frac{2}{3}}\right)
$$

by using Theorem 2.2, because $\sum_{0<n \leq \frac{3}{2}}\left((u v)^{2-\frac{2}{3} n}+(u v)^{\frac{2}{3} n}\right)=(u v)^{\frac{4}{3}}+(u v)^{\frac{2}{3}}$.

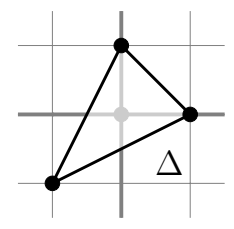

(a) Reflexive polygon.

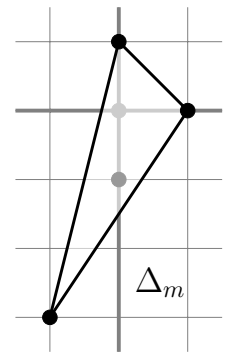

(b) $L D P$-polygon (here: $\mathrm{m}=3$ ).

Figure 4. Reflexive polygons and $L D P$-polygons.

Let $\Delta \subseteq N_{\mathbb{R}}$ be a 3-dimensional canonical Fano polytope, $\Sigma_{\Delta}$ the associated spanning fan of $\Delta$, and $X:=X_{\Delta}$ the corresponding toric Fano threefold with at worst canonical singularities. We are interested in a similar combinatorial formula to compute the stringy $E$-function $E_{\text {str }}(X ; u, v)$ of such a toric Fano threefold $X$ by making solely use of information about the lattice polytope $\Delta$ that yields the appearing coefficients $\psi_{\alpha}\left(\Sigma_{\Delta}\right)$ and rational powers of $(u v)^{\alpha}$ in $E_{\text {str }}(X ; u, v)$ in a direct way. To be precise, the following formula depends primarily on the volumes $v(\theta)$ and lattice distances $n_{\theta}$ to the origin of 2-dimensional faces $\theta \preceq \Delta$ :

Theorem C. Let $\Delta \subseteq N_{\mathbb{R}}$ be a 3-dimensional canonical Fano polytope. Then the stringy E-function of the corresponding canonical toric Fano threefold $X:=X_{\Delta}$ can be computed as

$$
E_{\text {str }}(X ; u, v)=\left((u v)^{3}+1\right)+r \cdot\left((u v)^{2}+(u v)\right)+\sum_{\substack{\theta \leq \Delta \\ \operatorname{dim}(\theta)=2, n_{\theta}>1}} v(\theta) \cdot\left(\sum_{k=1}^{n_{\theta}-1}(u v)^{\frac{k}{n_{\theta}}+1}\right)
$$

with $r:=|\Delta \cap N|-4$.

Proof. Applying Proposition 2.1, we know that the stringy $E$-function of $X$ is given as

$$
E_{\text {str }}(X ; u, v)=\sum_{\sigma \in \Sigma_{\Delta}^{\prime}}(u v-1)^{3-\operatorname{dim}(\sigma)} \sum_{n \in \square_{\sigma}^{\circ} \cap N}(u v)^{\operatorname{dim}(\sigma)+\kappa_{\Delta}(n)},
$$

where $\Sigma_{\Delta}^{\prime}$ is a simplicial subdivision of the fan $\Sigma_{\Delta}$ such that $\Sigma_{\Delta}(1) \subseteq \Sigma_{\Delta}^{\prime}(1)$ with $\left|\Sigma_{\Delta}^{\prime}(1)\right|=|\Delta \cap N|-1$, where the fan $\Sigma_{\Delta}^{\prime}$ is obtained by a triangulation of all 2dimensional faces $\theta \preceq \Delta$ of $\Delta$ and $\square_{\sigma}^{\circ}=\sigma^{\circ} \cap \square_{\sigma}$ for a cone $\sigma \in \Sigma_{\Delta}^{\prime}$. 
In each 1-dimensional cone $\rho \in \Sigma_{\Delta}^{\prime}(1)$, there exists exactly one lattice point $n \in$ $\square_{\rho}^{\circ} \cap N$ with $\kappa_{\Delta}(n)=-1$, because $n$ is a boundary lattice point of $\Delta$. Each 2dimensional cone $\tau \in \Sigma_{\Delta}^{\prime}(2)$ contains also exactly one lattice point $n \in \square_{\tau}^{\circ} \cap N$ with $\kappa_{\Delta}(n)=-2$, because the minimal lattice generators $u_{\rho^{\prime}}$ and $u_{\rho^{\prime \prime}}$ of $\tau$ are linearly independent over $\mathbb{R}$ with $n=u_{\rho^{\prime}}+u_{\rho^{\prime \prime}}$. These two facts yield

$$
\begin{aligned}
E_{\text {str }}(X ; u, v) & =(u v-1)^{3}+\sum_{\rho \in \Sigma_{\Delta}^{\prime}(1)}(u v-1)^{2}+\sum_{\tau \in \Sigma_{\Delta}^{\prime}(2)}(u v-1) \\
& +\sum_{\sigma \in \Sigma_{\Delta}^{\prime}(3)}\left(1+\sum_{\substack{n \in \square_{\sigma}^{\circ} \cap N \\
\kappa_{\Delta}(n) \in \mathbb{Q} \backslash \mathbb{Z}}}(u v)^{3+\kappa_{\Delta}(n)}\right),
\end{aligned}
$$

because each 3-dimensional cone $\sigma \in \Sigma_{\Delta}^{\prime}(3)$ has also exactly one lattice point $n \in \square_{\sigma}^{\circ} \cap$ $N$ with $\kappa_{\Delta}(n)=-3$. The intersections of all cones $\sigma \in \Sigma_{\Delta}^{\prime}$ with the 2-dimensional sphere $S^{2}$ defines a triangulation of $S^{2}$. Therefore, we have $\left|\Sigma_{\Delta}^{\prime}(3)\right|=2 \cdot\left|\Sigma_{\Delta}^{\prime}(1)\right|-4$ and $\left|\Sigma_{\Delta}^{\prime}(2)\right|=3 \cdot\left|\Sigma_{\Delta}^{\prime}(1)\right|-6$. Using $\left|\Sigma_{\Delta}^{\prime}(1)\right|=|\Delta \cap N|-1$, we get

$$
\begin{aligned}
E_{\text {str }}(X ; u, v) & =(u v-1)^{3}+(|\Delta \cap N|-1)(u v-1)^{2}+\sum_{\sigma \in \Sigma_{\Delta}^{\prime}(2)}(u v-1)+T+\sum_{\sigma \in \Sigma_{\Delta}^{\prime}(3)} 1 \\
(2) & =\left((u v)^{3}+1\right)+(|\Delta \cap N|-4)\left((u v)^{2}+(u v)\right)+T,
\end{aligned}
$$

where

$$
T:=\sum_{\sigma \in \Sigma_{\Delta}^{\prime}(3)} T_{\sigma} \text { and } T_{\sigma}:=\sum_{\substack{\left.n \in \square g \cap N \\ \kappa_{\Delta}(n) \in \mathbb{Q}\right) \mathbb{Z}}}(u v)^{3+\kappa_{\Delta}(n)} \text { for } \sigma \in \Sigma_{\Delta}^{\prime}(3)
$$

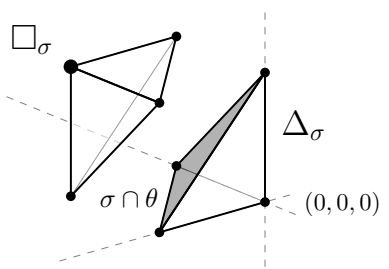

(a)

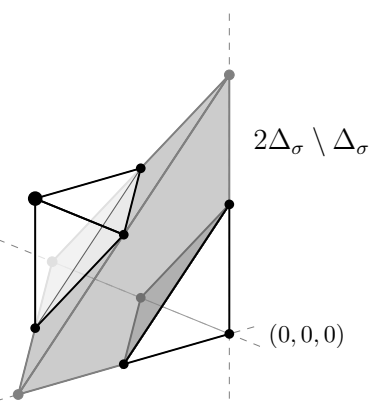

(b)

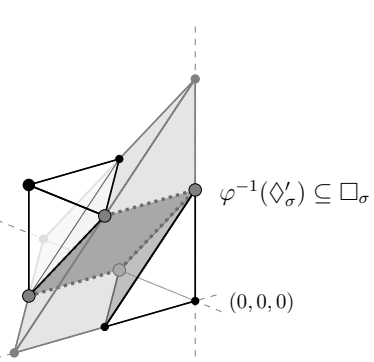

(c)

FiguRE 5. Illustration to detect $n \in \square_{\sigma}^{\circ} \cap N$ with $\kappa_{\Delta}(n) \in \mathbb{Q} \backslash \mathbb{Z}$.

Let $\sigma \in \Sigma_{\Delta}^{\prime}(3)$ be a 3-dimensional cone. Then $\sigma \cap \theta$ is a 2-dimensional simplex with $v(\sigma \cap \theta)=1$ contained in a 2-dimensional face $\theta \preceq \Delta$ of $\Delta$. The only lattice points of the 3-dimensional lattice simplex $\Delta_{\sigma}:=\sigma \cap \Delta$ are the origin $0 \in N$ and the three vertices $\nu_{1}, \nu_{2}$, and $\nu_{3}$ of the triangle $\sigma \cap \theta$ (Figure $5(\mathrm{a})$ ), because $\Delta$ is a canonical Fano polytope. Therefore, we get $v\left(\Delta_{\sigma}\right)=v(\sigma \cap \theta) \cdot n_{\theta}=n_{\theta}$.

If $n_{\theta}=1$, then $v\left(\Delta_{\sigma}\right)=1$ and $\sigma$ is generated by a basis of $N$, i.e., $T_{\sigma}=0$. For the following considerations, let $n_{\theta}$ be greater than 1. A lattice point $n \in \square_{\sigma}^{\circ} \cap N$ 
can not be contained in $\Delta_{\sigma}$ and $-\Delta_{\sigma}+\left(\nu_{1}+\nu_{2}+\nu_{3}\right)$ (Figure $\left.5(\mathrm{a})\right)$ as well as not in one of the three simplices $\Delta_{\sigma}+\nu_{1}, \Delta_{\sigma}+\nu_{2}$, and $\Delta_{\sigma}+\nu_{3}$ (Figure 5(b)). Therefore, $n$ belongs to $\left(2 \Delta_{\sigma}\right)^{\circ} \cap N$, i.e.,

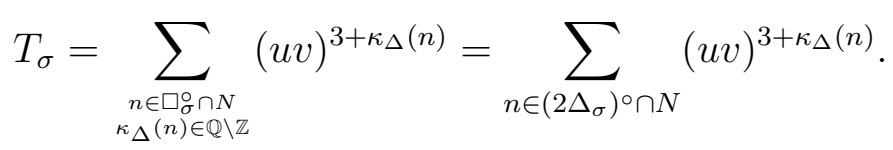

To compute $T_{\sigma}$, we apply the Theorem of White [Whi64], which claims that $\Delta_{\sigma}$ is affine unimodular isomorphic to a lattice simplex $\Delta_{\sigma}^{\prime}:=\operatorname{conv}\left(\nu_{1}^{\prime}, \nu_{2}^{\prime}, \nu_{3}^{\prime}, \nu_{4}^{\prime}\right)$ such that $\nu_{1}^{\prime}:=(0,0,0), \nu_{2}^{\prime}:=(1,0,0), \nu_{3}^{\prime}:=(0,0,1)$, and $\nu_{4}^{\prime}:=\left(a, n_{\theta}, 1\right)$ for some integer $a$ with $\operatorname{gcd}\left(a, n_{\theta}\right)=1$ (Figure 6(a)). In particular, $\Delta_{\sigma}^{\prime}$ is contained between the two planes $\{z=0\}$ and $\{z=1\}$. The affine isomorphism $\varphi: \Delta_{\sigma} \rightarrow \Delta_{\sigma}^{\prime}$ of White maps the vertex $0 \in \Delta_{\sigma}$ to the vertex $\nu_{4}^{\prime} \in \Delta_{\sigma}^{\prime}$ and transforms the linear function $\kappa_{\Delta}$ on $\sigma$ into the affine linear function $-1+\frac{y}{n_{\theta}}$ on a cone with origin $\nu_{4}^{\prime}$ and generaters $\nu_{i}^{\prime}-\nu_{4}^{\prime}$ $(1 \leq i \leq 3)$.

The double simplex $2 \Delta_{\sigma}^{\prime}$ is contained between the two planes $\{z=0\}$ and $\{z=$ $2\}$. Therefore, an interior lattice point $n \in\left(2 \Delta_{\sigma}^{\prime}\right)^{\circ}$ belongs to an interior point of $\diamond_{\sigma}^{\prime}:=2 \Delta_{\sigma}^{\prime} \cap\{z=1\}$, which is a parallelogram spanned by the vectors $(0,1,1)$ and $\left(a, n_{\theta}, 1\right)$ (Figure 6(b)). We remark that the isomorphism of White maps $\diamond_{\sigma}^{\prime}$ into a subset of $\square_{\sigma}$ (Figure $5(\mathrm{c})$ ).

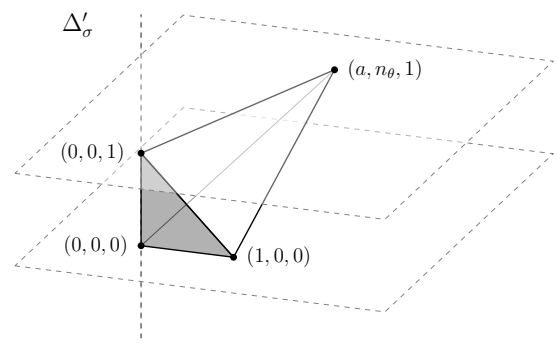

(a)

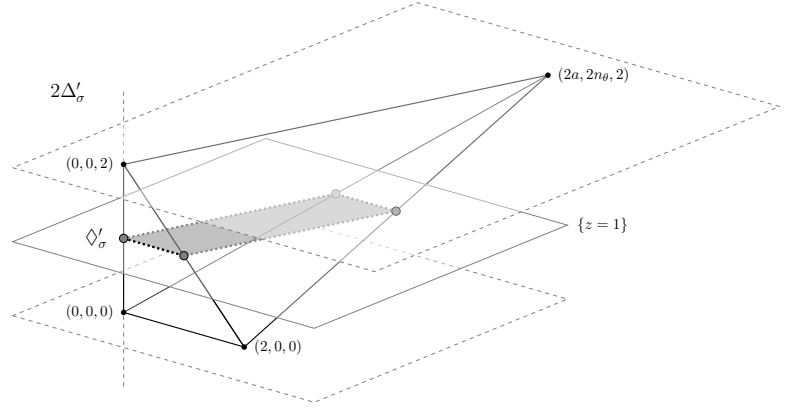

(b)

Figure 6 . Theorem of White (here: $a=1$ and $n_{\theta}=3$ ).

The volume of the parallelogram $\diamond_{\sigma}^{\prime}$ equals $n_{\theta}$ and it has exactly 4 boundary lattice points. By Pick's Theorem, there exist exactly $n_{\theta}-1$ lattice points in the interior of $\mho_{\sigma}^{\prime}$. We claim that the affine linear function $-1+\frac{y}{n_{\theta}}$ takes $n_{\theta}-1$ different values on these lattice points (Figure 7). Assume that there exist two lattice points $P=\left(P_{x}, P_{y}\right)$ and $P^{\prime}=\left(P_{x}^{\prime}, P_{y}^{\prime}\right)$ with $P_{x}^{\prime} \geq P_{x}$ in the interior of $\diamond_{\sigma}^{\prime}$ with the same affine linear function values, i.e., $P_{y}=P_{y}^{\prime}$ and $v\left(\operatorname{conv}\left(P, P^{\prime}\right)\right) \geq 1$. Set $E:=\operatorname{conv}((0,0),(1,0))$, then $P^{\prime}-P \in E^{\circ} \cap N=\emptyset$. Contradiction.

Therefore, we get the set equality

$$
\left\{\kappa_{\Delta}(n) \in \mathbb{Q} \backslash \mathbb{Z} \mid n \in \square_{\sigma}^{\circ} \cap N\right\}=\left\{-1-\frac{l}{n_{\theta}} \mid l \in\left\{1, \ldots, n_{\theta}-1\right\}\right\}
$$




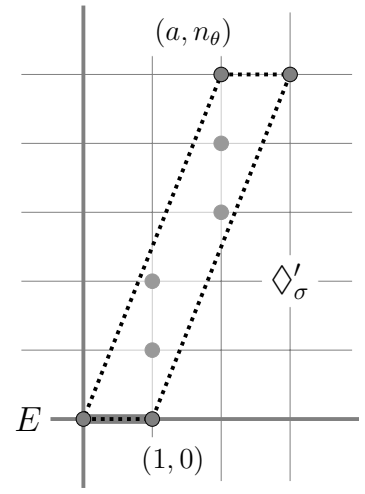

FiguRE 7. Illustration of $\diamond_{\sigma}^{\prime}$ (here: $a=2$ and $n_{\theta}=5$ ).

and Equation (3) can be rewritten in the form

$$
T_{\sigma}=\sum_{\substack{n \in \square \in \cap \\ \kappa_{\Delta}(n) \in \mathbb{Q} \backslash \mathbb{Z}}}(u v)^{3+\kappa_{\Delta}(n)}=\sum_{l=1}^{n_{\theta}-1}(u v)^{2-\frac{l}{n_{\theta}}}=\sum_{k=1}^{n_{\theta}-1}(u v)^{1+\frac{k}{n_{\theta}}} .
$$

Using this, the remaining last term in Equation (1) can be computed as

$$
T=\sum_{\sigma \in \Sigma_{\Delta}^{\prime}(3)} \sum_{\substack{n \in \square \circ \cap N \\ \kappa_{\Delta}(n) \in \mathbb{Q} \backslash \mathbb{Z}}}(u v)^{3+\kappa_{\Delta}(n)}=\sum_{\substack{\theta \preceq \Delta \\ \operatorname{dim}(\theta)=2, n_{\theta}>1}} v(\theta) \cdot\left(\sum_{k=1}^{n_{\theta}-1}(u v)^{\frac{k}{n_{\theta}}+1}\right),
$$

because $\sum_{\sigma \cap \theta \subseteq \theta} v(\sigma \cap \theta)=\sum_{\sigma \cap \theta \subseteq \theta} 1=v(\theta)$.

A combination of Equation (1), (2), and (4) yields the desired result

$$
E_{\text {str }}(X ; u, v)=\left((u v)^{3}+1\right)+r \cdot\left((u v)^{2}+(u v)\right)+\sum_{\substack{\theta \preceq \Delta \\ \operatorname{dim}(\theta)=2, n_{\theta}>1}} v(\theta) \cdot\left(\sum_{k=1}^{n_{\theta}-1}(u v)^{\frac{k}{n_{\theta}}+1}\right)
$$

with $r=|\Delta \cap N|-4$.

Example 2.4. Consider $\Delta_{1}=\operatorname{conv}\left(e_{1}, e_{2}, e_{3},-e_{1}-e_{2}-e_{3}\right), \Delta_{2}=\operatorname{conv}\left(e_{1}, e_{2}, e_{3},-e_{1}-\right.$ $\left.e_{2}-2 e_{3}\right)$, and $\Delta_{3}=\operatorname{conv}\left(e_{1}, e_{2}, e_{3},-5 e_{1}-6 e_{2}-8 e_{3}\right)$ (Figure 1) with $\Delta_{i}^{\circ} \cap N=\{0\}$ $(1 \leq i \leq 3)$. Therefore, $X_{\Delta_{i}}(1 \leq i \leq 3)$ are toric Fano threefolds with at worst canonical singularities with $X_{\Delta_{1}}=\mathbb{P}(1,1,1,1)=\mathbb{P}_{3}, X_{\Delta_{2}}=\mathbb{P}(1,1,1,2)$, and $X_{\Delta_{3}}=\mathbb{P}(1,5,6,8)$. By using Theorem $\mathrm{C}$ the associated stringy $E$-functions are given as

$$
\begin{gathered}
E_{\mathrm{str}}\left(X_{\Delta_{1}} ; u, v\right)=(u v)^{3}+(u v)^{2}+(u v)+1, \\
E_{\mathrm{str}}\left(X_{\Delta_{2}} ; u, v\right)=(u v)^{3}+(u v)^{2}+(u v)^{\frac{3}{2}}+(u v)+1,
\end{gathered}
$$

and

$$
\begin{aligned}
E_{\mathrm{str}}\left(X_{\Delta_{3}} ; u, v\right)= & (u v)^{3}+5 \cdot(u v)^{2}+2 \cdot(u v)^{\frac{5}{3}}+4 \cdot(u v)^{\frac{3}{2}} \\
& +2 \cdot(u v)^{\frac{4}{3}}+5 \cdot(u v)+1 .
\end{aligned}
$$




\section{The number 24 And 3-Dimensional CAnOnical Fano polytopes}

The lattice simplex $\Delta_{3}=\operatorname{conv}\left(e_{1}, e_{2}, e_{3},-5 e_{1}-6 e_{2}-8 e_{3}\right)$ (Figure 8(a) and 1(c)) is one of the simplest examples of a 3-dimensional canonical Fano polytope, which is not almost reflexive. The canonical Fano polytope $\Delta_{3}$ corresponds to the canonical toric Fano threefold $X_{\Delta_{3}}$, which is the weighted projective space $\mathbb{P}(1,5,6,8)$. It was observed by Corti and Golyshev [CG11] that an affine hypersurface $Z_{\Delta_{3}} \subseteq\left(\mathbb{C}^{*}\right)^{3}$ defined by a general Laurent polynomial $f_{\Delta_{3}} \in \mathbb{C}\left[x_{1}^{ \pm 1}, x_{2}^{ \pm 1}, x_{3}^{ \pm 1}\right]$ with the Newton polytope $\Delta_{3}$ is birational to an elliptic surface of Kodaira dimension one. In particular, $Z_{\Delta_{3}}$ is not birational to a $K 3$-surface. The dual polytope $\Delta_{3}^{*}$ (Figure $8(\mathrm{~b})$ ) is the rational simplex

$$
\operatorname{conv}((3,-1,-1),(-1,7 / 3,-1),(-1,-1,3 / 2),(-1,-1,-1)) .
$$

The two rational vertices of $\Delta_{3}^{*}$ are dual to two 2-dimensional faces of $\Delta_{3}$ (highlighted in gray and lightgray in Figure 8(a)) having lattice distance 2 and 3 to the origin, so that one gets

$$
\sum_{\substack{\theta \preceq \Delta_{3} \\ \operatorname{dim}(\theta)=2}} \frac{1}{n_{\theta}} \cdot v(\theta)=\frac{1}{2} \cdot 4+\frac{1}{3} \cdot 2+1 \cdot 1+1 \cdot 5=\frac{26}{3}
$$

and

$$
\sum_{\substack{\theta \preceq \Delta_{3} \\ \operatorname{dim}(\theta)=1}} v(\theta) \cdot v\left(\theta^{*}\right)=2 \cdot \frac{5}{6}+1 \cdot \frac{5}{2}+1 \cdot \frac{1}{2}+1 \cdot \frac{10}{3}+1 \cdot \frac{2}{3}+1 \cdot 4=\frac{38}{3} .
$$

Therefore, we obtain the equality

$$
v\left(\Delta_{3}\right)-\sum_{\substack{\theta \leq \Delta_{3} \\ \operatorname{dim}(\theta)=2}} \frac{1}{n_{\theta}} \cdot v(\theta)+\sum_{\substack{\theta \leq \Delta_{3} \\ \operatorname{dim}(\theta)=1}} v(\theta) \cdot v\left(\theta^{*}\right)=24
$$

because $v\left(\Delta_{3}\right)=20$.

As we have already mentioned, there exist exactly 9,089 canonical Fano polytopes in dimension 3, which are not almost reflexive. Our purpose is now to show that these polytopes also satisfy the identity of Theorem A, so that one gets the further generalization stated in

Theorem B. Let $\Delta \subseteq N_{\mathbb{R}}$ be an arbitrary 3-dimensional canonical Fano polytope. Then

$$
24=v(\Delta)-\sum_{\substack{\theta \preceq \Delta \\ \operatorname{dim}(\theta)=2}} \frac{1}{n_{\theta}} \cdot v(\theta)+\sum_{\substack{\theta \preceq \Delta \\ \operatorname{dim}(\theta)=1}} v(\theta) \cdot v\left(\theta^{*}\right) .
$$

To proof this statement, we can not use the ideas of Section 1, because there exist 9,089 canonical Fano polytopes $\Delta$ of dimension 3, that are not related to $K 3$-surfaces. In contrast, we use a completely different idea based on the stringy Libgober-Wood identity and its combinatorial interpretation for toric varieties containing generalized stringy Hodge numbers and intersection products of stringy Chern classes. 


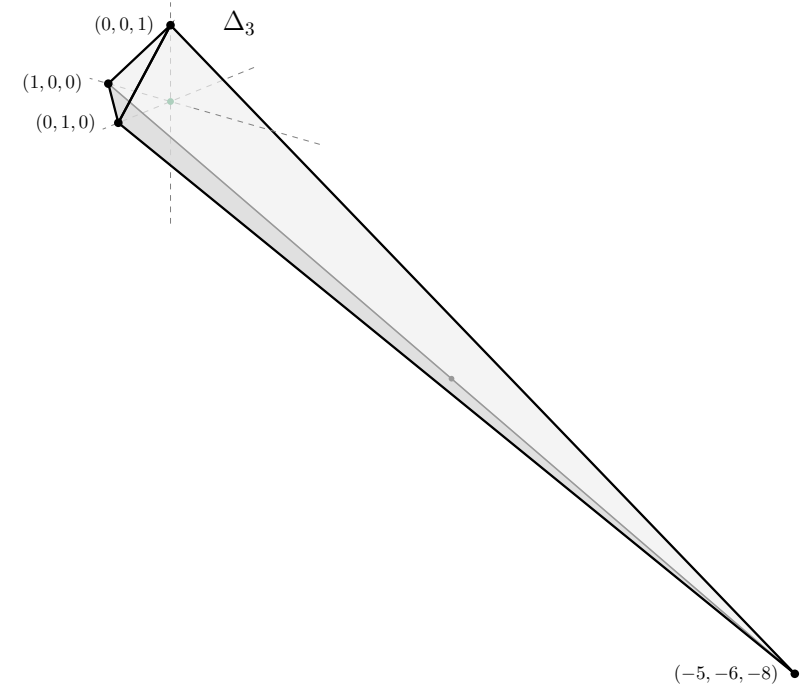

(a)

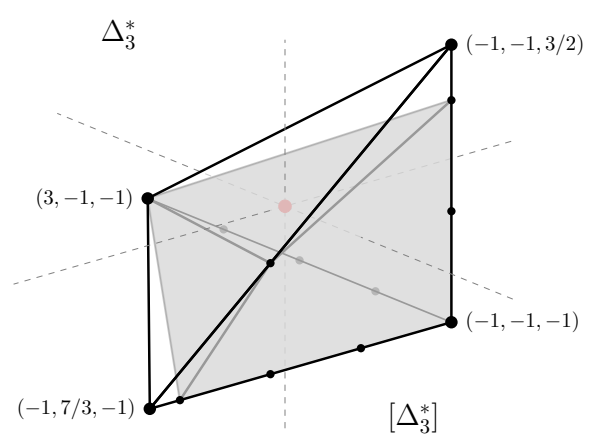

(b)

FiguRE 8. Illustration of a not almost reflexive polytope.

The original Libgober-Wood identity for smooth projective varieties has appeared in [LW90] and it has been generalized to the stringy Libgober-Wood identity

$$
\left.\frac{d^{2}}{d u^{2}} E_{\mathrm{str}}(X ; u, 1)\right|_{u=1}=\frac{3 d^{2}-5 d}{12} c_{d}^{\mathrm{str}}(X)+\frac{1}{6} c_{1}(X) \cdot c_{d-1}^{\mathrm{str}}(X)
$$

for a $d$-dimensional projective variety $X$ with at worst log-terminal singularities [Bat00, Theorem 3.8], where $c_{k}^{\operatorname{str}}(X) \in A^{k}(X)_{\mathbb{Q}}$ denotes the $k$-th stringy Chern class of $X$ [BS17, Section 1].

If $X=X_{\Sigma}$ is a $d$-dimensional $\mathbb{Q}$-Gorenstein projective toric variety of Gorenstein index $q_{X}$ associated with a $d$-dimensional fan $\Sigma$ of rational polyhedral cones in $N_{\mathbb{R}}$ and $-K_{X}$ an ample $\mathbb{Q}$-Cartier divisor, then the stringy $E$-function of $X$ can be written as a finite sum

$$
E_{\mathrm{str}}\left(X_{\Sigma} ; u, v\right)=\sum_{\alpha \in[0, d] \cap \frac{1}{q_{X}} \mathbb{Z}} \psi_{\alpha}(\Sigma)(u v)^{\alpha}
$$

[BS17, Proposition 4.1], where the coefficients $\psi_{\alpha}(\Sigma)$ are nonnegative integers (generalized stringy Hodge numbers) satisfying the conditions $\psi_{0}(\Sigma)=\psi_{d}(\Sigma)=1$ and $\psi_{\alpha}(\Sigma)=\psi_{d-\alpha}(\Sigma)$ for all $\alpha \in[0, d] \cap \frac{1}{q_{X}} \mathbb{Z}$ and the Gorenstein index $q_{X}$ is the smallest positive integer such that $q_{X} K_{X}$ is a Cartier divisor. Moreover, $\Delta_{-K_{X}} \subseteq M_{\mathbb{R}}$ denotes the $d$-dimensional rational polytope corresponding to the anticanonical divisor $-K_{X}$ of $X$ defined as

$$
\Delta_{-K_{X}}:=\left\{y \in M_{\mathbb{R}} \mid\left\langle y, u_{\rho}\right\rangle \geq-1 \forall \rho \in \Sigma_{\Delta}(1)\right\} \subseteq M_{\mathbb{R}}
$$


and $\Delta_{-K_{X}}^{\sigma} \preceq \Delta_{-K_{X}}$ a $k$-dimensional face of $\Delta_{-K_{X}}$ corresponding to a cone $\sigma \in$ $\Sigma(d-k)$ defined as

$$
\Delta_{-K_{X}}^{\sigma}:=\left\{y \in \Delta_{-K_{X}} \mid\left\langle y, u_{\rho}\right\rangle=-1 \forall \rho \in \Sigma_{\Delta}(1) \text { with } \rho \in \sigma\right\}
$$

[BS17, Theorem 3.5], where $u_{\rho} \in N$ denotes the primitive ray generator of a 1dimensional cone $\rho \in \Sigma_{\Delta}(1)$.

In this setting, the stringy Libgober-Wood identity is equivalent to the combinatorial identity stated in

Proposition 3.1 ([BS17, Theorem 4.3]). Let $X:=X_{\Sigma}$ be a d-dimensional $\mathbb{Q}$ Gorenstein projective toric variety of Gorenstein index $q_{X}$ associated with a fan $\Sigma$ of rational polyhedral cones in $N_{\mathbb{R}}$ and $-K_{X}$ an ample $\mathbb{Q}$-Cartier divisor. Then

$$
\sum_{\alpha \in[0, d] \cap \frac{1}{q_{X}} \mathbb{Z}} \psi_{\alpha}(\Sigma)\left(\alpha-\frac{d}{2}\right)^{2}=\frac{d}{12} v(\Sigma)+\frac{1}{6} \sum_{\sigma \in \Sigma(d-1)} v(\sigma) \cdot v\left(\Delta_{-K_{X}}^{\sigma}\right),
$$

where $v(\Sigma):=\sum_{\sigma \in \Sigma(d)} v(\sigma)$ denotes the normalized volume of a fan $\Sigma$ and $v(\sigma)$ the normalized volume of a cone $\sigma$ defined as the normalized volume of the polytope obtained as the convex hull of the origin and all primitive ray generators of the cone $\sigma \in \Sigma$.

Proof of Theorem B. We apply Proposition 3.1 to the canonical toric Fano threefold $X:=X_{\Delta}$ with at worst canonical singularities corresponding to the canonical Fano polytope $\Delta$ of dimension 3. In this case, the associated fan $\Sigma_{\Delta}$ is the spanning fan of $\Delta$, i.e., it consists of cones $\sigma_{\theta}=\mathbb{R}_{\geq 0} \theta$, where $\theta \preceq \Delta$ runs over all faces $\theta$ of $\Delta$.

By construction of the fan $\Sigma_{\Delta}$, it is easy to show that $v\left(\Sigma_{\Delta}\right)=v(\Delta)$. Moreover,

$$
v\left(\sigma_{\theta}\right)=v(\theta)
$$

for any 1-dimensional face $\theta \preceq \Delta$, because $v\left(\sigma_{\theta}\right)=v(\operatorname{conv}(0, \theta))=v\left(h_{\theta}\right) \cdot v(\theta)$, where $h_{\theta}$ denotes the height of the lattice triangle $\operatorname{conv}(0, \theta)$ with base $\theta$ and $v\left(h_{\theta}\right)=1$, because all nonzero lattice points of the lattice triangle $\operatorname{conv}(0, \theta)$ are contained in its side $\theta$.

By definition of the dual polytope $\Delta^{*}$, every 1-dimensional face $\theta^{*}$ of $\Delta^{*}$ has lattice distance 1 to the origin. So one has $\Delta_{-K_{X}}=\Delta^{*}$ and $\Delta_{-K_{X}}^{\sigma_{\theta}}=\theta^{*}$ yielding

$$
v\left(\Delta_{-K_{X}}^{\sigma_{\theta}}\right)=v\left(\theta^{*}\right)
$$

Therefore, the right hand side of the stringy Libgober-Wood identity in Proposition 3.1 equals

$$
\frac{3}{12} v(\Delta)+\frac{1}{6} \sum_{\substack{\theta \preceq \Delta \\ \operatorname{dim}(\theta)=1}} v(\theta) \cdot v\left(\theta^{*}\right)
$$

and the number $v(\Delta)$ equals

$$
E_{\text {str }}(X ; 1,1)=2+2 \cdot(|\Delta \cap N|-4)+\sum_{\substack{\theta \preceq \Delta \\ \operatorname{dim}(\theta)=2, n_{\theta}>1}} v(\theta) \cdot \sum_{k=1}^{n_{\theta}-1} 1
$$


[Bat98, Proposition 4.10]. The left hand side of the stringy Libgober-Wood identity in Proposition 3.1 can be computed by Theorem C as

$$
2 \cdot 1\left(\frac{3}{2}\right)^{2}+2 \cdot(|\Delta \cap N|-4)\left(1-\frac{3}{2}\right)^{2}+\sum_{\substack{\theta \preceq \Delta \\ \operatorname{dim}(\theta)=2, n_{\theta}>1}} v(\theta) \cdot \sum_{k=1}^{n_{\theta}-1} 1 \cdot\left(\frac{k}{n_{\theta}}-\frac{1}{2}\right)^{2} .
$$

Comparing right and left hand sides, a short calculation yields

$$
24=6 \cdot \sum_{\substack{\theta \subseteq \Delta \\ \operatorname{dim}(\theta)=2, n_{\theta}>1}} v(\theta) \cdot \sum_{k=1}^{n_{\theta}-1}\left(\frac{1}{4}-\left(\frac{k}{n_{\theta}}-\frac{1}{2}\right)^{2}\right)+\sum_{\substack{\theta \preceq \Delta \\ \operatorname{dim}(\theta)=1}} v(\theta) \cdot v\left(\theta^{*}\right)
$$

and using Lemma 3.2 from below adds up to

$$
\begin{aligned}
24 & =\sum_{\substack{\theta \preceq \Delta \\
\operatorname{dim}(\theta)=2, n_{\theta}>1}} v(\theta)\left(n_{\theta}-\frac{1}{n_{\theta}}\right)+\sum_{\substack{\theta \preceq \Delta \\
\operatorname{dim}(\theta)=1}} v(\theta) \cdot v\left(\theta^{*}\right) \\
\Leftrightarrow 24 & =v(\Delta)-\sum_{\substack{\theta \preceq \Delta \\
\operatorname{dim}(\theta)=2}} \frac{1}{n_{\theta}} \cdot v(\theta)+\sum_{\substack{\theta \preceq \Delta \\
\operatorname{dim}(\theta)=1}} v(\theta) \cdot v\left(\theta^{*}\right),
\end{aligned}
$$

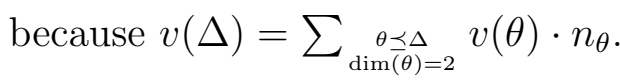

Lemma 3.2. Let $n$ be a positive integer. Then

$$
6 \cdot \sum_{k=1}^{n-1}\left(\frac{1}{4}-\left(\frac{k}{n}-\frac{1}{2}\right)^{2}\right)=n-\frac{1}{n}
$$

Proof. Applying the well-known formulas for $\sum_{k=1}^{n-1} k$ and $\sum_{k=1}^{n-1} k^{2}$, we get

$$
\begin{gathered}
6 \cdot \sum_{k=1}^{n-1}\left(\frac{1}{4}-\left(\frac{k}{n}-\frac{1}{2}\right)^{2}\right)=6 \cdot \sum_{k=1}^{n-1}\left(\frac{1}{4}-\frac{k^{2}}{n^{2}}+\frac{k}{n}-\frac{1}{4}\right)=-\frac{6}{n^{2}} \cdot \sum_{k=1}^{n-1} k^{2}+\frac{6}{n} \sum_{k=1}^{n-1} k \\
=-\frac{1}{n^{2}}[(n-1) n(2(n-1)+1)]+\frac{3}{n}[(n-1) n]=n-\frac{1}{n} .
\end{gathered}
$$

\section{REFERENCES}

[Bat94] Victor V. Batyrev, Dual Polyhedra and Mirror Symmetry for Calabi-Yau Hypersurfaces in Toric Varieties, Journal of Algebraic Geometry 3 (1994), no. 3, 493-535. 1, 2

[Bat98]_ _ Stringy Hodge numbers of varieties with Gorenstein canonical singularities, Integrable systems and algebraic geometry (Kobe/Kyoto 1997) (1998), 1-31, World Sci. Publ., River Edge, NJ. 4, 5, 18

[Bat00] _ Stringy Hodge Numbers and Virasoro Algebra, Mathematical Research Letters 7 (2000), no. 1-2, 155-164. 16

[Bat17]_ The stringy Euler number of Calabi-Yau hypersurfaces in toric varieties and the Mavlyutov duality, arXiv:1707.02602v2. 3, 6, 7

$\left[\mathrm{BCF}^{+} 05\right]$ Matthias Beck, Beifang Chen, Lenny Fukshansky, Christian Haase, Allen Knutson, Bruce Reznick, Sinai Robins, and Achill Schürmann, Problems from the Cottonwood Room, Integer points in polyhedra - geometry, number theory, algebra, optimization, Contemp. Math., vol. 374, Amer. Math. Soc., Providence, RI, 2005, pp. 179-191. 2, 8 
[BD96] Victor V. Batyrev and Dimitrios I. Dais, Strong McKay correspondende, string-theoretic Hodge numbers and mirror symmetry, Topology 35 (1996), no. 4, 901-929. 2

[BS17] Victor V. Batyrev and Karin Schaller, Stringy Chern classes of singular toric varieties and their applications, Communications in Number Theory and Physics 11 (2017), 1-40. 4, 10, 16, 17

[CG11] Alessio Corti and Vasily Golyshev, Hypergeometric Equations and Weighted Projective Spaces, Science China Mathematics 54 (2011), no. 8, 1577-1590. 15

[Dan78] Vladimir I. Danilov, The geometry of toric varieties, Uspekhi Mat. Nauk 33 (1978), no. 2(200), 85-134, 247. 1

[DHVW85] Lance J. Dixon, Jeffrey A. Harvey, Cumrun Vafa, and Edward Witten, Strings on orbifolds, Nuclear Physics B 261 (1985), 678-686. 4

[Kas10] Alexander M. Kasprzyk, Canonical toric Fano threefolds, Canadian Journal of Mathematics 62 (2010), no. 6, 1293-1309. 1, 2, 3

[Kho78] Askold G. Khovanskiu, Newton polyhedra and the genus of complete intersections, Funktsional. Anal. i Prilozhen. 12 (1978), no. 1, 51-61. 3

[KKN10] Alexander M. Kasprzyk, Maximilian Kreuzer, and Benjamin Nill, On the combinatorial classification of toric log del Pezzo surfaces, LMS J. Comput. Math. 13 (2010), 33-46. 10

[KS98] Maximilian Kreuzer and Harald Skarke, Classification of Reflexive Polyhedra in Three Dimensions, Advances in Theoretical and Mathematical Physics 2 (1998), no. 4, 853871. 2

[LW90] Anatoly S. Libgober and John W. Wood, Uniqueness of the complex structure on Kähler manifolds of certain homotopy types, Journal of Differential Geometry 32 (1990), 139154. 16

[Rei83] Miles Reid, Minimal models of canonical 3-folds, Algebraic varieties and analytic varieties (Tokyo, 1981), Adv. Stud. Pure Math., vol. 1, North-Holland, Amsterdam, 1983, pp. $131-180.1$

[Ska96] Harald Skarke, Weight systems for toric Calabi-Yau varieties and reflexivity of Newton polyhedra, Modern Phys. Lett. A 11 (1996), no. 20, 1637-1652. 6

[Whi64] George K. White, Lattice tetrahedra, Canad. J. Math. 16 (1964), 389-396. 13

Mathematisches Institut, Universität Tübingen, Auf Der Morgenstelle 10, 72076 Tübingen, Germany

E-mail address: victor.batyrev@uni-tuebingen.de

Mathematisches Institut, Universität Tübingen, Auf der Morgenstelle 10, 72076 TüBIngEn, GERMAnY

E-mail address: karin.schaller@uni-tuebingen.de 